\title{
CINÉTICA DE SECAGEM E COEFICIENTE DE DIFUSIVIDADE DE PIMENTAS MALAGUETAS DESIDRATADAS EM SECADOR DE LEITO FIXO
}

\author{
K. T. CATELAM ${ }^{1}$, M.M.N. NAKAMURA ${ }^{1}$ e J. TELIS-ROMERO ${ }^{1}$ \\ ${ }^{1}$ Universidade Estadual Paulista Júlio de Mesquita Filho (UNESP), Departamento de Engenharia \\ e Tecnologia de Alimentos \\ E-mail para contato: kelly_tfcl@yahoo.com.br
}

\begin{abstract}
RESUMO - Apesar de todas as vantagens do processo de secagem de alimentos, a secagem de pimentas ainda é pouco explorada. Realizou-se a secagem de pimentas malaguetas em secador de leito fixo a $40,50,60$ e $70^{\circ} \mathrm{C}$ por 5 horas com velocidade do ar de $1,6 \mathrm{~m} / \mathrm{s}$ e pesagens a cada 10 minutos para construção das curvas de taxa de secagem em função do tempo. Na modelagem destas curvas empregaram-se os modelos empíricos de Henderson-Pabis, Lewis, Page e o modelo teórico difusivo obtido da segunda Lei de Fick considerando geometria de cilindro finito. A taxa de secagem aumentou com o incremento da temperatura, conforme esperado. Na relação entre taxa de secagem e umidade da pimenta, observou-se apenas o período de taxa de secagem decrescente com a diminuição da umidade, resultado comum à grande maioria dos alimentos já estudados na literatura. $\mathrm{O}$ melhor ajuste foi obtido com o modelo de Page, enquanto que, com o modelo difusivo, encontraram-se valores de difusividade da ordem de $10^{-10}$ e $10^{-11} \mathrm{~m}^{2} / \mathrm{s}$.
\end{abstract}

\section{INTRODUÇÃO}

A pimenta malagueta (Capsicum frutescens) é um fruto alongado, ereto, com cerca de 2 $\mathrm{cm}$ de comprimento, $0,5 \mathrm{~cm}$ de largura e coloração vermelha forte (maduros) ou verdes (imaturos). Sua pungência varia de média a alta e seu aroma é baixo (Embrapa, 2013). O mercado para a industrialização da pimenta consiste, basicamente, na secagem, na conserva do fruto inteiro e na produção de molho (Fraife Filho et al., 2012). O processo de secagem de produtos alimentícios tem sido objeto de especial interesse na indústria de alimentos, por apresentar vantagens como: abaixamento da atividade de água, inibição do desenvolvimento de microrganismos, garantindo a conservação do produto, proteção contra degradação enzimática e oxidativa, redução da massa e disponibilidade do produto durante qualquer época do ano. Permite também o transporte e o armazenamento sem a cadeia do frio (CanoChauca et al., 2005). Além disto, a perda de massa que ocorre quando um alimento é seco, diminui consideravelmente os custos de transporte e manuseio. Apesar de todas estas vantagens, a secagem de pimentas ainda é pouco explorada.

O agronegócio da pimenta (Capsicum spp) desidratada no Brasil está relacionado aos produtos oriundos de agricultores familiares da região de Turuçu-RS. A quase totalidade da pimenta produzida neste município é seca através de sistema natural. A duração do processo 
de secagem varia com a intensidade solar e dos ventos. Entretanto, por falta de desenvolvimento tecnológico apropriado e disponibilidade de recursos para investimentos em equipamentos que sejam adequados à desidratação artificial da matéria-prima, elevadas perdas (ao redor de 40\%) são registradas anualmente, e o produto apresenta baixa qualidade. Devido ao alto tempo de secagem ( 3 a 7 dias) muitos frutos apodrecem ou fermentam. Por este motivo, a Embrapa elaborou em 2005 um Manual para Processamento de Pimentas (Capsicum spp) Desidratadas com os procedimentos básicos para secagem de pimentas em secadores de cabine a $60^{\circ} \mathrm{C}$ até não se visualizar mais umidade na amostra. Este manual inclui os procedimentos de higiene e limpeza para preparo da pimenta, sendo uma alternativa para elaboração comercial de pimentas desidratadas em flocos. A desidratação de pimentas para fabricação de conservas vem sendo informalmente utilizada por produtores para tentar aumentar a durabilidade das mesmas, obtendo-se resultados positivos. Alguns estudos têm visado a redução do tempo de secagem ou aumento da qualidade das pimentas usando secadores melhores que o solar (Chandy et al., 1992), porém, os modelos matemáticos relacionados à secagem de pimentas ainda são bastante escassos.

A secagem convencional por ar aquecido é realizada em secadores cujo sistema baseiase na circulação de ar quente, combinando, dessa forma, transferência de calor e de massa. $\mathrm{O}$ alimento a ser seco é disposto em bandejas que permitem a passagem do ar aquecido no interior do secador com baixa umidade relativa. Com este tipo de processo pode-se controlar variáveis, como: temperatura (T), porcentagem de umidade relativa do ar (UR), velocidade e direção de fluxo de ar (V). Considerando a pimenta malagueta, foram realizados estudos de Santos et al. (2012), onde avaliou-se a cinética de secagem de pimentas malaguetas cultivadas no estado de Sergipe através da variação de temperatura de 50 a $110^{\circ} \mathrm{C}$, com intervalo de 10 ${ }^{\circ} \mathrm{C}$ entre os ensaios. No Brasil, alguns autores estudaram a cinética de secagem de outras espécies de pimentas, como Carneiro (2011) que trabalhou com pimenta dedo-de-moça nas temperaturas de 40,50 e $60{ }^{\circ} \mathrm{C}$ e Ponte et al. (2009) que realizou seus ensaios com pimenta de cheiro em secador de bandejas a 50,60 e $70^{\circ} \mathrm{C}$.

O processo de secagem de um produto, sob condições constantes de T, UR e V, pode ser dividido em um período de velocidade constante e outro de velocidade decrescente (Hall, 1980; Foust et al., 2008). De acordo com Queiroz et al. (1985) são dois os métodos comumente usados para analisar a secagem de produtos biológicos no período a taxa decrescente: o empírico e o teórico. O método empírico consiste em formar grupos físicos adimensionais que podem ser facilmente investigados por experimentos de laboratório e baseia-se nas condições externas, como T, UR e V. Entretanto, não fornece indicação sobre o transporte de energia e de água no interior dos produtos. Atualmente, são empregados muitos modelos empíricos para as análises de curvas de secagem, como os modelos de Page, Lewis e Henderson-Pabis, mostrados nas Equações 1 a 3, respectivamente.

$$
\begin{aligned}
& M=\frac{X-X_{e}}{X_{i}-X_{e}}=\exp \left(-k t^{N}\right) \\
& M=\frac{X-X_{e}}{X_{i}-X_{e}}=\exp (-k t) \\
& M=\frac{X-X_{e}}{X_{i}-X_{e}}=\alpha \cdot \exp (-k t)
\end{aligned}
$$


onde: $\mathrm{M}$ é a razão de umidade (adimensional), $\mathrm{X}(\mathrm{kg} / \mathrm{kg}, \mathrm{bs})$ é a umidade do produto no tempo $\mathrm{t}, \mathrm{X}_{\mathrm{i}}$ é o conteúdo de umidade inicial $\left(\mathrm{kg} / \mathrm{kg}\right.$, bs), $\mathrm{X}_{\mathrm{e}}$ é o conteúdo de umidade de equilíbrio $(\mathrm{kg} / \mathrm{kg}, \mathrm{bs}), \mathrm{k}$ são as constantes de velocidade de secagem $\left(\mathrm{s}^{-1}\right), \mathrm{N}$ e $\alpha$ são constantes.

Os métodos teóricos usados para descrever o processo de secagem são baseados em leis físicas que tentam explicar o mecanismo de transferência de água. A principal teoria utilizada para interpretar a secagem de alimentos e de produtos agrícolas é a teoria da difusão da umidade como líquido ou vapor, representada pela segunda lei de Fick (Crank, 1975). Os modelos que empregam a teoria da difusão podem descrever de forma aceitável o perfil da distribuição de água no interior de determinado produto agrícola desde que seja possível correlacionar sua forma à geometria de um sólido perfeito, além da exigência do estabelecimento de uma relação funcional entre o coeficiente de difusão, o teor de umidade e a temperatura. As soluções analíticas da lei de Fick para uma placa infinita e cilindro infinito são mostradas nas Equações 4, 4a e 5, respectivamente. Multiplicando-se os resultados obtidos nas Equações 4 e 5, obtém-se o resultado para cilindro finito.

$$
M_{(x, t) L}=\frac{X-X_{e}}{X_{i}-X_{e}}=2 \sum_{n=0}^{\infty} \frac{(-1)^{n}}{\lambda_{n} L} e^{\left(-\alpha \lambda_{n}^{2} t\right)} \cos \left(\lambda_{n} x\right)
$$

onde:

$$
\begin{aligned}
& \lambda_{n} L=(2 n+1) \frac{\pi}{2} \\
& M_{(r, t) C}=\frac{X-X_{e}}{X_{i}-X_{e}}=2 \sum_{n=1}^{\infty} \frac{1}{\lambda_{n} R J_{1}\left(\lambda_{n} R\right)} e^{\left(-\alpha \lambda_{n}{ }^{2} t\right)} \mathrm{J}_{0}\left(\lambda_{n} r\right)
\end{aligned}
$$

onde $\alpha=$ difusividade aparente $\left(\mathrm{m}^{2} / \mathrm{s}\right), \mathrm{L}=$ espessura média do produto $(\mathrm{m}), \mathrm{R}=$ raio equivalente do cilindro $(\mathrm{m}), \mathrm{t}=$ tempo de secagem $(\mathrm{s}), \mathrm{J}_{0}$ e $\mathrm{J}_{1}=$ funções de Bessel de primeira espécie e ordem 0 e 1, respectivamente, $\mathrm{n}=$ número de séries na solução analítica $(\mathrm{n}=20), \mathrm{r}=$ posição $(\mathrm{m}) ; \lambda_{\mathrm{n}}=$ raízes da função de Bessel na equação (5) tal que $\mathrm{J}_{0}\left(\lambda_{n} R\right)=0$.

\section{OBJETIVOS}

Determinar a cinética de secagem da pimenta malagueta a 40,50, 60 e $70^{\circ} \mathrm{C}$ através da secagem em secador de leito fixo com velocidade do ar constante, aplicando os modelos empíricos de Page, Henderson-Pabis e Lewis, além de calcular o coeficiente de difusão da pimenta utilizando a solução analítica da segunda lei de Fick para geometria de cilindro finito.

\section{MATERIAL E MÉTODOS}

Utilizaram-se pimentas malaguetas (Capsicum frutescens), compradas no mercado local de São José do Rio Preto - SP, com uso imediato à compra.

\subsection{Dimensões da Pimenta}


Mediram-se comprimento (C), diâmetro inferior (DI), diâmetro superior (DS) e maior diâmetro (DM) de dez amostras de pimentas malaguetas in natura com paquímetro. Calcularam-se o diâmetro equivalente $(\Phi)$, a esfericidade $(\varepsilon)$ e a relação entre o comprimento e o maior diâmetro (C/DM), conforme mostram as equações 6 e 7 (Foust et al., 2008).

$$
\begin{gathered}
\phi=(D I . D M \cdot D S)^{(1 / 3)} \\
\varepsilon=\frac{\phi}{D M}
\end{gathered}
$$

\subsection{Ensaios de Secagem, Cinética de Secagem e Coeficiente de Difusão}

Os ensaios de secagem foram realizados em secador de leito fixo com as variáveis do processo monitoradas manualmente. As secagens convectivas das pimentas foram realizadas

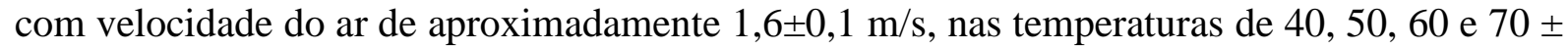
$1^{\circ} \mathrm{C}$ por 5 horas. As amostras eram pesadas na bandeja a cada 10 minutos, em balança semianalítica, e os teores de umidade (base seca) inicial e final foram determinados pelo método da estufa a $105^{\circ} \mathrm{C}$ por 24 horas. Mediram-se também a umidade relativa do ar de secagem e do ar ambiente, assim como a temperatura deste último. A taxa de secagem (R) das pimentas malaguetas nas 4 temperaturas de secagem foi calculada, aproximadamente, conforme equação 8.

$$
R=-\frac{d X}{d t}
$$

onde: $\mathrm{X}=$ umidade em base seca $(\mathrm{bs}) ; \mathrm{t}=$ tempo de secagem (s).

Plotou-se o gráfico de taxa de secagem versus umidade (bs) obtida ao longo das 5 horas de secagem para avaliação do período de secagem constante e decrescente, a fim de escolher as melhores equações para a determinação da cinética de secagem das pimentas nas 4 temperaturas de secagem. Através de pesagens periódicas das bandejas contendo o produto, determinaram-se curvas de umidade versus tempo (curvas de secagem). Na modelagem das curvas de secagem para as pimentas, foram empregados os modelos empíricos de HendersonPabis (equação 3), Lewis (equação 2), Page (equação 1) e o modelo teórico difusivo (derivado da solução analítica da segunda lei de Fick) para geometria de cilindro finito. As constantes empíricas destes modelos e a difusividade através do modelo difusivo foram determinadas experimentalmente através das curvas de secagem conforme ensaios do item 3.2.

\section{RESULTADOS E DISCUSSÃO}

\subsection{Dimensões das Pimentas}

As dimensões das pimentas são mostrados na Tabela 1. Pelo valor da esfericidade média $(0,60 \pm 0,08 \mathrm{~cm})$ conclui-se que trata-se de aproximação para um cilindro. Já a partir da relação 
comprimento sobre maior diâmetro, verifica-se que trata-se de um cilindro com comprimento

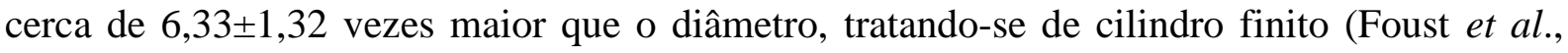
2008).

Tabela 1 - Média e desvio padrão (dp) do comprimento (C), diâmetro superior (DS), maior diâmetro $(\mathrm{DM})$, diâmetro inferior (DI), diâmetro equivalente $(\Phi)$, esfericidade $(\varepsilon)$, relação comprimento/maior diâmetro (C/DM) das amostras de pimenta malagueta in natura

\begin{tabular}{cccccccc}
\hline & $\mathbf{C}(\mathbf{c m})$ & DS $(\mathbf{c m})$ & DM $(\mathbf{c m})$ & DI $(\mathbf{c m})$ & $\mathbf{\Phi}(\mathbf{c m})$ & $\boldsymbol{\varepsilon}$ & C/DM \\
\hline Média & 2,33 & 0,36 & 0,62 & 0,23 & 0,37 & 0,60 & 6,33 \\
$\mathbf{d p}$ & 0,56 & 0,06 & 0,14 & 0,05 & 0,06 & 0,08 & 1,32 \\
\hline
\end{tabular}

\subsection{Ensaios no secador de leito fixo e Modelagem da Cinética de Secagem}

A Tabela 2 mostra os parâmetros de cada um dos ensaios de secagem realizados.

Tabela 2 - Temperaturas (T) e umidades relativas (UR) dos ensaios de secagem

\begin{tabular}{cccccccc}
\hline $\begin{array}{c}\text { T ar } \\
\text { secagem }\end{array}$ & $\begin{array}{c}\text { UR ar } \\
\text { secagem }\end{array}$ & $\begin{array}{c}\mathrm{T} \\
\text { ambiente }\end{array}$ & $\begin{array}{c}\text { UR } \\
\text { ambiente }\end{array}$ & $\begin{array}{c}\text { T ar } \\
\text { secagem }\end{array}$ & $\begin{array}{c}\text { UR ar } \\
\text { secagem }\end{array}$ & $\begin{array}{c}\mathrm{T} \\
\text { ambiente }\end{array}$ & $\begin{array}{c}\text { UR } \\
\text { ambiente }\end{array}$ \\
\hline $\mathbf{4 0}^{\circ} \mathbf{C}$ & $32 \%$ & $29,5^{\circ} \mathrm{C}$ & $47,1 \%$ & $\mathbf{6 0}^{\circ} \mathbf{C}$ & $13 \%$ & $30,9^{\circ} \mathrm{C}$ & $27,0 \%$ \\
$\mathbf{5 0}^{\circ} \mathbf{C}$ & $16 \%$ & $30,7^{\circ} \mathrm{C}$ & $27,0 \%$ & $\mathbf{7 0}^{\circ} \mathbf{C}$ & $7,5 \%$ & $29,1^{\circ} \mathrm{C}$ & $52,3 \%$ \\
\hline
\end{tabular}

A Figura 1a mostra o gráfico do teor de umidade em base seca em função do tempo de secagem. Observa-se uma maior rapidez na queda de umidade (aumento da taxa de secagem) com o aumento da temperatura de secagem. Nos ensaios a 60 e $70^{\circ} \mathrm{C}$ é possível observar uma tendência à umidade de equilíbrio, pois a curva passa a ter valores quase constantes de umidade, indicando que este tempo de secagem juntamente com estas condições de temperatura, velocidade do ar e umidade relativa podem ser suficientes para uma secagem ideal da amostra. A Figura 1b mostra a taxa de secagem das pimentas nas 4 temperaturas avaliadas. Observa-se pela Figura $1 \mathrm{~b}$ que todas as taxas de secagem são decrescentes, não sendo observado período de taxa constante de secagem, indicando assim que o principal mecanismo de migração da água do interior do produto para sua superfície é a difusão. Hall (1980) explica que o período de taxa decrescente inicia quando a migração interna de umidade passa a controlar o processo, em outras palavras, a velocidade de transferência de massa no interior do sólido é menor que a taxa de evaporação na superfície. A ausência do período de taxa constante, que ocorre com a grande maioria das frutas e alguns vegetais, pode ser explicada pela natureza da ligação da água do produto. Embora exista água superficial livre, ela encontra-se em suspensão com outros constituintes do produto, como por exemplo, os açúcares (Keey, 1975). Santos et al. (2012) também observaram apenas o comportamento decrescente para curvas de taxa de secagem de pimenta malagueta de 50 a $110^{\circ} \mathrm{C}$.

A taxa é maior na temperatura de $70^{\circ} \mathrm{C}$, seguindo pela de 60,50 e $40^{\circ} \mathrm{C}$, logo, tem-se que a elevação da temperatura resultou num efeito positivo sobre a taxa de secagem. A cinética de secagem está diretamente relacionada com a temperatura do processo, o aumento na temperatura resulta em maior transferência de umidade. À altas temperaturas a UR do ar de secagem é menor e, devido a isto, a diferença na pressão de vapor parcial entre as pimentas e 
a alta temperatura do ar é maior comparada à diferença entre a pressão parcial de vapor entre as pimentas e o ar a baixa temperatura. Logo, a taxa de secagem é maior à altas temperaturas de secagem (Kaleemullah e Kailappan, 2005).

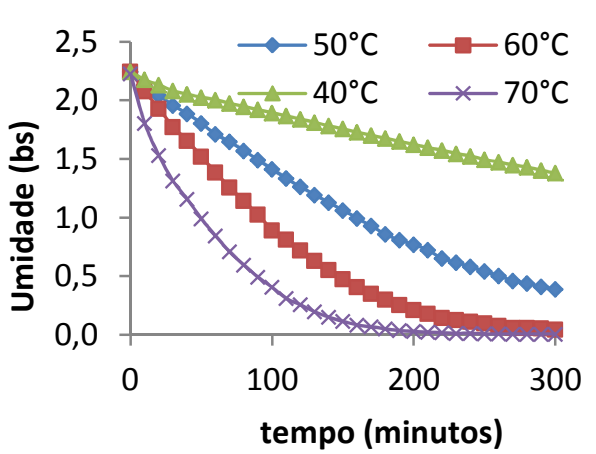

(a)

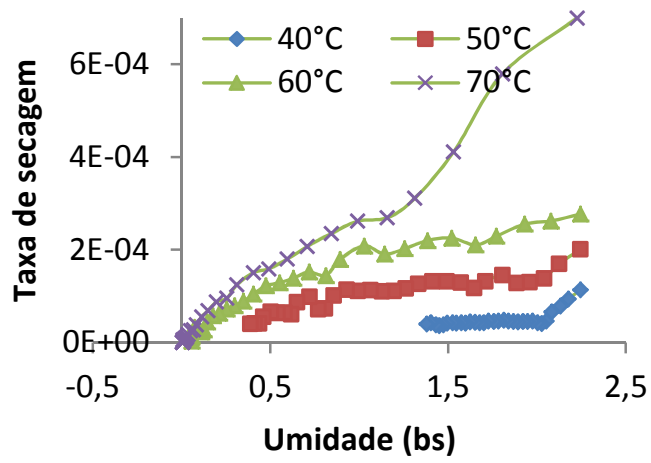

(b)

Figura 1 - (a) Teor de umidade da pimenta malagueta em função do tempo de secagem; (b)

Taxa de secagem das pimentas malaguetas em função da umidade.

Pode-se visualizar também a influência do conteúdo de umidade, sendo a taxa de secagem maior em altos teores de umidade, como esperado. À medida que o teor de umidade decresce, decresce também a taxa de secagem, até se aproximar a zero. Isso se deve a natureza da ligação da água com a matriz do produto. Em altos percentuais de água, esta se encontra em suspensão com componentes como os açúcares, sendo removida com maior facilidade. Com o decorrer do tempo, ela passa a ser encontrada fortemente ligada à matriz sólida do produto, cuja dificuldade de remoção é maior resultando em baixas taxas de secagem.

A Tabela 3 mostra os dados utilizados para modelagem da cinética de secagem pelos modelos de Page, Henderson-Pabis, Lewis e difusivo. A Tabela 4 mostra os valores das constantes de cada modelo, assim como coeficiente de correlação $\left(\mathrm{R}^{2}\right)$ e erro médio relativo (MRE).

Tabela 3 - Dados experimentais empregados na modelagem das curvas de secagem para pimentas malaguetas às temperaturas de $40,50,60$ e $70^{\circ} \mathrm{C}$ e velocidade do ar de $1,6 \mathrm{~m} / \mathrm{s}$

\begin{tabular}{cccccccccc}
\hline $\mathbf{T}\left({ }^{\circ} \mathbf{C}\right)$ & UR & aw & Xeq (bs) & Xi (bs) & T $\left({ }^{\circ} \mathbf{C}\right)$ & UR & aw & Xeq (bs) & Xi (bs) \\
\hline $\mathbf{4 0}$ & $32 \%$ & 0,32 & 0,0594 & 2,2446 & $\mathbf{6 0}$ & $13 \%$ & 0,13 & 0,0300 & 2,2446 \\
$\mathbf{5 0}$ & $16 \%$ & 0,16 & 0,0375 & 2,2446 & $\mathbf{7 0}$ & $7,5 \%$ & 0,075 & 0,0079 & 2,2258 \\
\hline
\end{tabular}

A Figura 2 mostra os modelos de Page, Lewis, Henderson-Pabis e difusivo ajustado às curvas de secagem de 40,50,60, e $70^{\circ} \mathrm{C}$. Comparando-se estas figuras, observa-se que o modelo que melhor se ajustou foi o de Page o qual, conforme Tabela 4, apresentou os maiores valores de $\mathrm{R}^{2}$. Foram obtidos bons ajustes para o modelo de Fick para cilindro finito conforme observa-se na Tabela $4\left(\mathrm{R}^{2}\right.$ acima de 0,92$)$, observando-se um aumento da difusividade com a temperatura. De acordo com Rizvi (2005) a difusividade depende da temperatura de secagem e da variedade e composição do produto, variando de valores da ordem de $10^{-9}$ a $10^{-11} \mathrm{~m}^{2} / \mathrm{s}$ para os alimentos. $\mathrm{O}$ aumento da temperatura de secagem causa a diminuição da viscosidade da água e, sendo essa viscosidade uma medida de resistência do fluido ao escoamento, essa 
diminuição pode causar alterações na difusão da água dentro do produto favorecendo a movimentação desse fluido no produto (Gonelli et al., 2010).

Tabela 4 - Constantes dos modelos de Page, Lewis, Henderson-Pabis e difusividade para a cinética de secagem das pimentas secas a $60^{\circ} \mathrm{C}$ em secador de leito fixo

\begin{tabular}{|c|c|c|c|c|c|}
\hline Modelo & Temperatura & $40^{\circ} \mathrm{C}$ & $50^{\circ} \mathrm{C}$ & $60^{\circ} \mathrm{C}$ & $\mathbf{7 0}^{\circ} \mathrm{C}$ \\
\hline \multirow{4}{*}{ Page } & $\mathbf{k}$ & $4,121 \times 10^{-5}$ & $1,026 \times 10^{-5}$ & $8,669 \times 10^{-6}$ & $1,122 \times 10^{-4}$ \\
\hline & $\mathbf{N}$ & 0,959 & 1,235 & 1,334 & 1,113 \\
\hline & & 0,998 & 0,999 & 0,998 & 0,997 \\
\hline & MRE & 0,617 & 1,899 & 17,881 & 83,298 \\
\hline \multirow{3}{*}{ Lewis } & $\mathbf{k}$ & $2,821 \times 10^{-5}$ & $8,967 \times 10^{-5}$ & $1,688 \times 10^{-4}$ & $2,930 \times 10^{-4}$ \\
\hline & $\mathbf{R}^{2}$ & 0,998 & 0,993 & 0,991 & 0,997 \\
\hline & MRE & 0,721 & 7,242 & 53,114 & 118,351 \\
\hline \multirow{4}{*}{$\begin{array}{l}\text { Henderson- } \\
\text { Pabis }\end{array}$} & $\alpha$ & 0,983 & 1,043 & 1,076 & 0,998 \\
\hline & $\mathbf{k}$ & $2,666 \times 10^{-5}$ & $9,455 \times 10^{-5}$ & $1,808 \times 10^{-4}$ & $2,925 \times 10^{-4}$ \\
\hline & $\mathbf{R}^{2}$ & 0,998 & 0,991 & 0,987 & 0,997 \\
\hline & MRE & 0,399 & 5,785 & 44,604 & 118,946 \\
\hline \multirow{3}{*}{ Difusivo } & $D_{\text {iff }}\left(\mathrm{m}^{2} / \mathrm{s}\right)$ & $3,666 \times 10^{-11}$ & $8,004 \times 10^{-11}$ & $1,425 \times 10^{-10}$ & $2,375 \times 10^{-10}$ \\
\hline & $\mathbf{R}^{2}$ & 0,924 & 0,987 & 0,988 & 0,977 \\
\hline & MRE & 8,551 & 7,775 & 19,041 & 39,644 \\
\hline
\end{tabular}
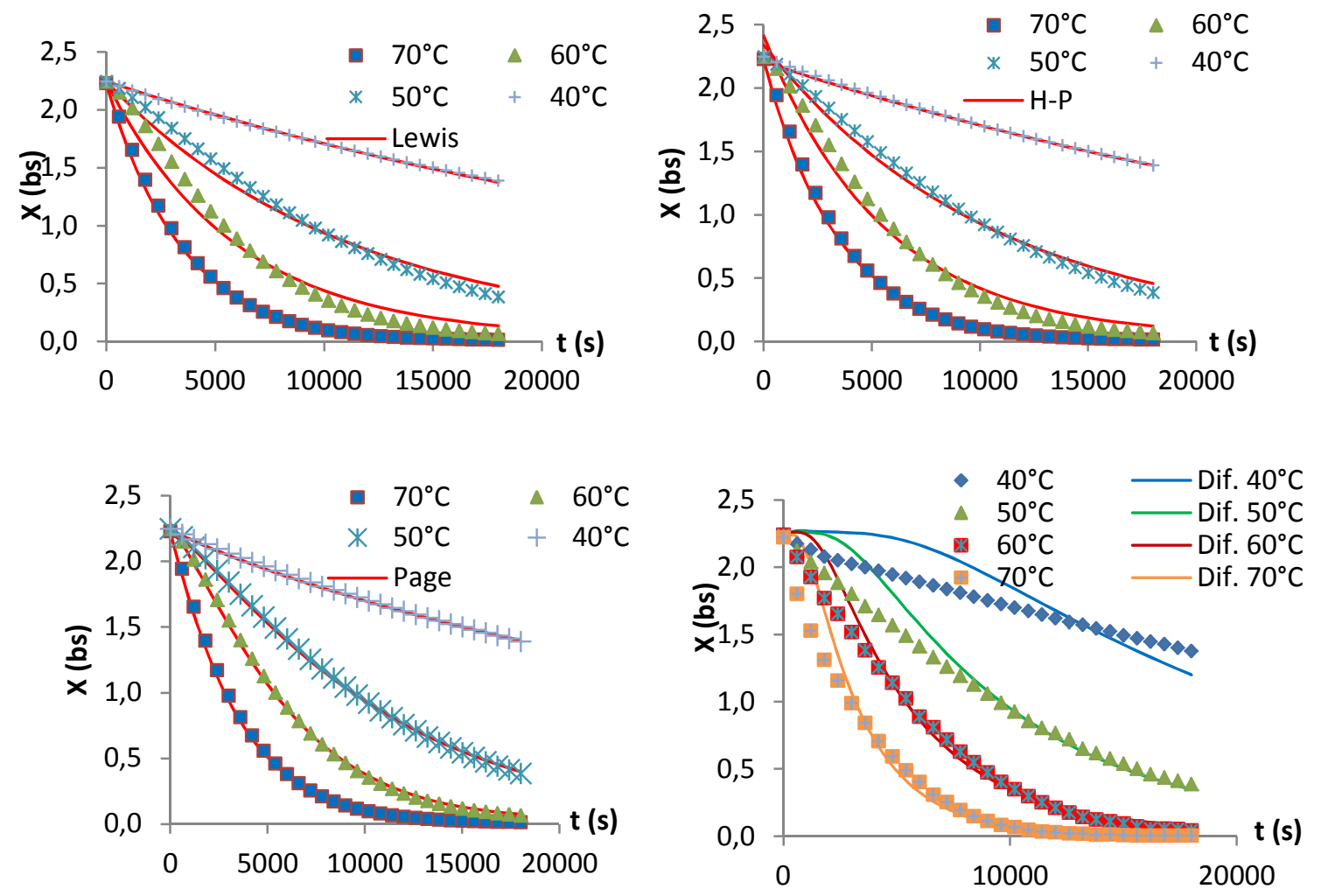

Figura 2 - Curvas de secagem com ajustes dos modelos de Lewis, Henderson-Pabis (H-P), Page e do modelo difusivo para cilindro finito. 


\section{CONCLUSÃO}

As curvas de secagem da pimenta malagueta nas temperaturas de $40,50,60$ e $70^{\circ} \mathrm{C}$ encontram-se dentro do esperado, mostrando uma maior taxa de secagem na temperatura de $70^{\circ} \mathrm{C}$. O modelo de Page foi o que melhor se ajustou aos dados experimentais de todas as temperaturas, enquanto o modelo difusivo mostrou valores de ordem de $10^{-11}$ e $10^{-10}$ para a difusividade nas pimentas, aumentando seu valor com a temperatura de secagem.

\section{REFERÊNCIAS}

CARNEIRO, P.A.V.; SILVA, F.S.; PORTO, A.G. Análise da cinética de secagem de pimenta variedade dedo de moça. In: Congresso de Iniciação Científica, 4ª . (JC), 2011, Cáceres/MT: Pró-Reitoria de Pesquisa e Pós-Graduação - PRPPG, 2011.

CHANDY, E.; ILYAS, S.M.; SAMUEL, D.V.K.; SINGH, A. Effect of some physical treatments on drying characteristics of red chillies. Proceedings of the International Agricultural Engineering Conference, Bangkok, Thailan, p. 489-498, 1992.

CRANK, J. The Mathematics of Diffusion. Oxford: Clarendon Press, 1975.

EMBRAPA HORTALIÇAS. Capsicum: Pimentas e Pimentões do Brasil. Disponível em: http://www.cnph.embrapa.br/capsicum/index.htm>. Acesso em: 23/06/2013.

FOUST, A.S.; WENZEL, L.A.; CLUMP, C.W.; ANDERSON, L.B. Princípios das Operações Unitárias. Rio de Janeiro: Guanabara Dois, 2008.

FRAIFE FILHO, G.A. Pimenta. Disponível em: <http://www.ceplac.gov.br/radar/pimenta.htm>. Acesso em: 02/07/2012.

GONELLI, A.L.D.; CORREA, P.C.; OLIVEIRA, G.H.H.; GOME,S C.F.; BOTELHO, F.M. Water sorption isotherms and thermodynamic properties of pearl millet grain. J. Food Sci. Technol, v.45, p. 828-838, 2010.

HALL, C. W. Drying and storage of agricultural crops. Westport: AVI Publishing Company, 1980.

KALEEMULlAH, S; KAILAPPAN, R. Drying Kinetics of Red Chillies in a Rotary Dryer. Biosyst. Eng., v.92, n.1, p.15-23, 2005.

KEEY, R. B. Drying: principles and practice. Oxford: Pergamon Press, 1975.

RIZVI, S. S. H. Thermodynamic properties of foods in dehydration. In: RAO, M. A.; RIZVI, S. S. H.; DATTA, A. K.: Engineering properties of foods. Boca Raton: Taylor \& Francis Group, 2005.

SANTOS, J.A.B.; SILVA, G.F.; PAGANI, A.A.C. Estudo da cinética de secagem da pimenta malagueta (Capsicum Spp) cultivada no estado de Sergipe. Revista GEINTEC, v. 2, n.5, p.465-471, 2012. 